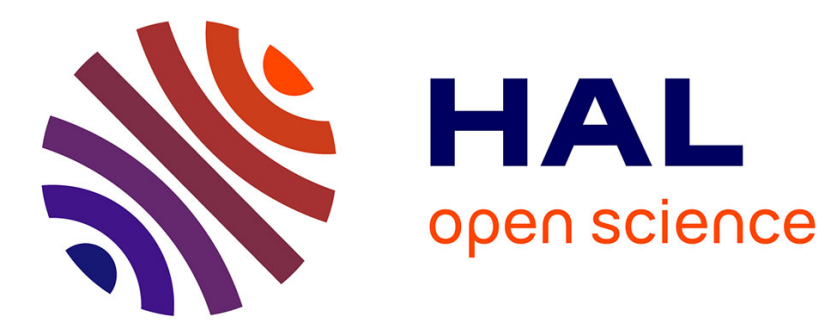

\title{
Polarized confocal theta microscopy
}

Olivier Haeberlé, Hiromitsu Furukawa, Koji Tenjimbayashi

\section{To cite this version:}

Olivier Haeberlé, Hiromitsu Furukawa, Koji Tenjimbayashi. Polarized confocal theta microscopy.

Comptes Rendus. Physique, 2002, 3, pp.1445-1450. 10.1016/S1631-0705(02)01406-8 . hal-00951612

\section{HAL Id: hal-00951612 \\ https://hal.science/hal-00951612}

Submitted on 25 Feb 2014

HAL is a multi-disciplinary open access archive for the deposit and dissemination of scientific research documents, whether they are published or not. The documents may come from teaching and research institutions in France or abroad, or from public or private research centers.
L'archive ouverte pluridisciplinaire HAL, est destinée au dépôt et à la diffusion de documents scientifiques de niveau recherche, publiés ou non, émanant des établissements d'enseignement et de recherche français ou étrangers, des laboratoires publics ou privés. 
Rubrique : Electromagnetism, Optics

\title{
Polarized confocal theta microscopy
}

\author{
Olivier HAEBERLÉ ${ }^{1}$, Hiromitsu FURUKAWA², Koji TENJIMBAYASHI² \\ ${ }^{1}$ Groupe LabEl - Laboratoire MIPS, Université de Haute-Alsace IUT Mulhouse, 61 rue A. Camus F- \\ 68093 Mulhouse Cedex France \\ ${ }^{2}$ OptMec, Photonics Research Institute, AIST, Namiki 1-2, Tsukuba, Ibaraki 305-8564 Japan
}

\begin{abstract}
We propose a comprehensive treatment of theta microscopy based on dipole emission, which describes better the fluorescence emission than the isotropic emission model, as fluorescence emission is often polarized. Formulas describing the point spread function for polarized confocal fluorescence theta microscopy are given. Examples are given and some advantages of polarized theta fluorescence microscopy are presented.
\end{abstract}

Fluorescence microscopy, Reflection microscopy, Theta microscopie

\section{Microscopie confocale theta polarisée}

Résumé

\begin{abstract}
Nous présentons un modèle pour la microscopie de fluorescence theta basé sur le rayonnement dipolaire, qui décrit mieux le phénomène de fluorescence que le modèle isotropique, car l'émission de fluorescence est souvent polarisée. Les formules décrivant la tache de diffraction pour la microscopie de fluorescence polarisée en montage theta sont données. Des exemples sont donnés, et certains avantages à utiliser la polarisation en microscopie de fluorescence theta sont présentés.
\end{abstract}

Microscopie de fluorescence, Microscopie en réflexion, Microscopie Theta

\section{Correspondance:}

Olivier Haeberlé

Adresse permanente:

Laboratoire LabEl, IUT de Mulhouse, 61 rue A. Camus 68093 Mulhouse Cedex

Tél : 0389337660 Fax : 0389337605

o.haeberle@uha.fr

du 15/04/02 au 26/08/02 :

OptMeC - The Photonic Research Institute,

AIST, Namiki 1-2, Tsukuba, 305-8564, JAPAN

tel:+81-298-61-7095 fax:+81-298-61-7091

o.haeberle@aist.go.jp 


\section{Version française abrégée}

En microscopie classique et confocale [1,2], un compromis entre résolution et distance de travail doit être accepté, les objectifs à grande distance de travail ayant une faible ouverture numérique, et donc une faible résolution. Le microscope theta a été proposé pour pallier à cet inconvénient. Il consiste à utiliser deux objectifs de microscope pour l'excitation et la détection, dont les axes optiques sont perpendiculaires [3]. La Figure 1 en décrit le principe. L'objectif 1 est utilisé pour illuminer le specimen, et la détection se fait au travers de l'objectif 2 .

Nous proposons un traitement détaillé du microscope theta, basé sur la théorie vectorielle de la diffraction pour les objectifs à grande ouverture numérique, et sur le modèle d'émission dipolaire.

La configuration du microscope theta oblige à utiliser des objectifs sans lamelle, la RIO d'excitation est donc calculée à l'aide du modèle de Richards et Wolf [1]. Les équations $(1,2)$ donnent le champ électromagnétique au point focal. La RIO du microscope theta est alors souvent calculée à l'aide de l'équation (3), en considérant une émission de fluorescence isotrope et non-polarisée [3]. L'émission de fluorescence étant polarisée [4,5], il est plus rigoureux de considérer le champ émis par un dipole [6,7]. Le moment et le champ dipolaires sont alors donnés par les équations (4) et (5). Le champ est capté par l'objectif 2, et refocalisé sur le détecteur. Avec un objectif de refocalisation de faible ouverture numérique, le détecteur quadratique enregistre alors le carré de la transformée de Fourier du champ électromagnétique: équation (7), les équations (6a) et (6b) décrivant le champ, calculé à l'aide des intégrales (8). La formule (9) lie les angles entre l'objectif de détection 2 et la lentille de refocalisation $[6,7]$.

Nous avons calculés les RIO pour un microscope theta utilisant des objectifs d'ouverture numérique N.A. $=0.8$ à immersion à eau [8], avec une excitation à $\lambda=400 \mathrm{~nm}$ et une détection $\lambda=450 \mathrm{~nm}$ (Cascade Blue from Molecular Probes). La Figure 2 montre les résultats obtenus. La Figure 2(a) montre la RIO calculée avec le modèle isotrope. Les Figures 2(b) et 2(c) montrent la RIO calculée avec le modèle dipolaire, pour une excitation x-polarisée et une détection x-polarisée et pour une excitation z-polarisée et une détection $\mathrm{x}$-polarisée, respectivement. Le modèle isotrope a tendance à surestimer la résolution en $\mathrm{x}$, et sous-estimer la résolution en z et en y. La Figure 3 montre les RIO pour une détection y-polarisée. Non seulement les formes des RIO sont très différentes, mais surtout l'intensité est environ 400x plus faible. Les RIOs montrent la forme caractéristique en feuille de trèfle aussi prévue en microscopie confocale polarisée $[6,7,9,11]$ (Figures 3(a) et 3(c)). Notons cependant que les RIO sont maintenant asymétriques, comme le montrent les Figures 3(b), 3(d) et 3(e), qui présentent des coupes $(x-y)$ et (x-z) à divers plans en profondeur et latéralement. 


\section{Introduction}

In classical and even confocal microscopy [1,2], a trade-off between resolution and working distance must always be done. Long working distance objectives have a low numerical aperture, and therefore a low resolution, especially along the optical axis. High numerical aperture objectives permit a high resolution, but at the price of a usually very short working distance.

Theta microscopy has been proposed as an effective solution to combine high-resolution with long working distances. Theta microscopy therefore provides the unique feature of allowing the study of large biological sample while keeping a good resolution.

In previous work, the Point Spread Function of theta microscopy was calculated using an isotropic emission model, with unpolarized excitation. However, fluorescence is known to be polarized. As a consequence, dipole emission describes better the fluorescence process.

We propose a comprehensive treatment of polarized fluorescence microscopy based on the electromagnetic theory of high numerical aperture objective, taking into account dipole emission by the fluorescent dye.

\section{Polarized Fluorescence Theta Microscopy}

The idea of theta microscopy is to diminish the size of the Point Spread Function by using two microscope objectives with crossed optical axes [3]. Doing so, the lower resolution along the optical axis of the detection objective is compensated by the fact that this axis corresponds to a lateral axis for the illumination objective. Figure 1 describes the considered setup. Objective 1 is used for fluorescence excitation, and objective 2 is for confocal detection.

This optical configuration does not allow to use glass slides, so, the Richard and Wolf model of diffraction describes well the illumination PSF formation [1]. Considering a plane wave, linearly polarized along the $\mathrm{x}$-axis, being focused by objective 1 , the electric field at point $\mathrm{P}$ with coordinates $\mathrm{x}=\mathrm{x}^{\prime}, \mathrm{y}=-\mathrm{z}^{\prime}, \mathrm{z}=\mathrm{y}{ }^{\prime}$ (see Fig. 1: ( $\left.\mathrm{x}, \mathrm{y}, \mathrm{z}\right)$ coordinates are for detection arm and $\left(\mathrm{x}^{\prime}, \mathrm{y}^{\prime}, \mathrm{z}^{\prime}\right)$ coordinates are for illumination arm) in the focal region is written as:

$$
E_{x}=-i\left(I_{0}+I_{2} \cos 2 \phi\right), E_{y}=2 I_{1} \cos \phi, E_{z}=-i\left(I_{2} \sin 2 \phi\right)
$$

with:

$$
\begin{aligned}
& I_{0}=\int_{0}^{\alpha} a(\theta) \sin \theta(1+\cos \theta) J_{0}\left(k \sqrt{x^{2}+z^{2}} \sin \theta\right) \exp (-i k y \cos \theta) d \theta \\
& I_{1}=\int_{0}^{\alpha} a(\theta) \sin ^{2} \theta J_{1}\left(k \sqrt{x^{2}+z^{2}} \sin \theta\right) \exp (-i k y \cos \theta) d \theta \\
& I_{2}=\int_{0}^{\alpha} a(\theta) \sin \theta(1-\cos \theta) J_{2}\left(k \sqrt{x^{2}+z^{2}} \sin \theta\right) \exp (-i k y \cos \theta) d \theta
\end{aligned}
$$

The apodization function in Eqs. (2) is given by $\mathrm{a}(\theta)=\cos ^{1 / 2} \theta, \phi$ the angle between the direction of observation and the $\mathrm{x}$-axis (see Figure 1), $\alpha$ is the objective lens aperture angle, $\mathrm{J}_{\mathrm{n}}$ is the Bessel function of first kind or order $\mathrm{n}$, and $\mathrm{k}=\mathrm{k}_{0} \cdot \mathrm{n}$ with $\mathrm{n}$ being the immersion medium refraction index.

The classical treatment of confocal or theta confocal microscopy consists in considering that the excited molecule acts as an isotropic radiator. The final Point Spread Function is simply calculated as the product of the illumination PSF by the detection PSF. For a theta setup, it is written as [3]:

$$
\operatorname{PSF}^{\text {theta }}(x, y, z)=\operatorname{PSF}(x, y, z) \times \operatorname{PSF}(-z, y, x)
$$

as the difference in wavelength between illumination and detection is often neglected for simplicity. In that approach, the illumination and detection PSF are computed using the Richards and Wolf model. However, this model is for a plane wave focused into the medium, thus valid to compute the illumination PSF, but calculating the detection PSF as an illumination PSF constitutes another approximation.

Furthermore, fluorescence is known to be polarized [4,5]. A more rigorous approach therefore consists in considering a point scatterer whose far field emission is that of an electric dipole, which moment $\mathbf{p}$ is proportional to the illumination field [6,7]. Hence, the Cartesian components of the dipole moment are 
given by:

$$
p_{x}=-i\left(I_{0}+I_{2} \cos 2 \phi\right), p_{y}=2 I_{1} \cos \phi, p_{z}=-i\left(I_{2} \sin 2 \phi\right)
$$

The electric field in the radiation zone being given by:

$$
\mathbf{E}_{d p}=-\mathbf{r} \times(\mathbf{r} \times \mathbf{p})
$$

The dipole electric vector is collimated by the detection objective, then passed through an analyzer and refocused onto the detector. Considering a low aperture detector lens, a quadratic detector therefore records the intensity $\mathrm{I}=\left|E_{\mathrm{d}}\right|^{2}$, with $E_{\mathrm{d}}$ being the Fourier transform of the scalar field [6]:

$$
\begin{aligned}
& E_{d x}=p_{x}\left(I_{0}+I_{2} \cos 2 \phi_{p}\right)+p_{y} I_{2} \sin 2 \phi_{p}-2 i p_{z} \cos \phi_{p} \\
& E_{d y}=p_{x} I_{2} \sin 2 \phi_{p}+p_{y}\left(I_{0}-I_{2} \cos 2 \phi_{p}\right)-2 i p_{z} \sin \phi_{p}
\end{aligned}
$$

and then one obtains the intensity PSF after the polarizer as [6]:

$$
\operatorname{PSF}(x, y, z)=\left|\cos ^{2} \gamma E_{x}+\sin \gamma \cos \gamma E_{y}\right|^{2}+\left|\cos \gamma \sin \gamma E_{x}+\sin ^{2} \gamma E_{y}\right|^{2}
$$

with:

$$
\begin{aligned}
& I_{0}^{d}=\int_{0}^{\alpha_{d}} \sqrt{\cos \theta_{1}} \sin 2 \theta_{d} J_{0}\left(k \rho \sin \theta_{d}\right)\left(1+\cos \theta_{1}\right) \exp \left(-i k z_{s} \cos \theta_{1}\right) d \theta_{d} \\
& I_{1}^{d}=\int_{0}^{\alpha_{d}} \sqrt{\cos \theta_{1}} \sin 2 \theta_{d} J_{1}\left(k \rho \sin \theta_{d}\right) \sin \theta_{1} \exp \left(-i k_{1} z_{s} \cos \theta_{1}\right) d \theta_{d} \\
& I_{2}^{d}=\int_{0}^{\alpha_{d}} \sqrt{\cos \theta_{1}} \sin 2 \theta_{d} J_{2}\left(k \rho \sin \theta_{d}\right)\left(1-\cos \theta_{1}\right) \exp \left(-i k_{1} z_{s} \cos \theta_{1}\right) d \theta_{d}
\end{aligned}
$$

$\gamma$ being the angle between the analyzer axis and the x-axis. The angular aperture of the detector lens $\alpha_{d}$ and the coordinate angle $\theta_{\mathrm{d}}$ are linked to the objective lens angular aperture $\alpha_{1}$ and the coordinate $\theta_{1}$ by the relation $[6,7]$ :

$$
\left(n_{1} \sin \alpha_{1}\right) /\left(n_{d} \sin \alpha_{d}\right)=\left(n_{1} \sin \theta_{1}\right) /\left(n_{d} \sin \theta_{d}\right)=\beta
$$

where $\beta$ is the nominal magnification of the objective lens, and $n_{1}$ and $n_{d}$ are the indices of refraction of the immersion medium and the image space, respectively [6,7].

\section{Exemples}

Figure 2 shows computed point spread functions. An Olympus Universal Plan Fluorite 40x waterimmersion objective with N.A. $=0.8$ is considered. This objective has a working distance of $3.3 \mathrm{~mm}$ and would be well suited for a theta setup, after a slight modification of the enclosure [8]. The excitation PSF is computed at $\lambda=400 \mathrm{~nm}$ and we consider emission at $\lambda=450 \mathrm{~nm}$ (corresponding to excitation and emission of Cascade Blue from Molecular Probes). The specimen is scanned through the focus. For the sake of simplicity, a point-like incoherent detector is considered [6,7]. Figure 2(a) shows the PSF computed using the classical isotropic emission model, for unpolarized excitation and detection, using Eq. (3). Figure 2(b) shows the PSF using the dipole model for x-polarized excitation and x-polarized detection. It is shown that the classical model tends to slightly overestimate the x-lateral resolution, while simultaneously slightly understimates the y-lateral and z-longitudinal resolution. The dipole emission models predicts a more isotropic PSF, with better separation of the lateral lobes (more noticeable differences are expected if larger N.A. objectives may be used [6,7,9]). The x-resolution is $220 \mathrm{~nm}$, the $\mathrm{y}$ and z- resolution being of $270 \mathrm{~nm}$ (versus $210 \mathrm{~nm}$ and $290 \mathrm{~nm}$ for the isotropic emission model).

An inherent problem of polarized detection in confocal microscopy is that the detection of molecules excited by light with z-axis polarization is limited by the fact that the excitation (and therefore the available signal) is much weaker than for polarization along the $\mathrm{x}$-axis or the $\mathrm{y}$-axis $[9,10]$. Typically the 
signal is 100 times lower [9]. A possible approach is to use phase filters to enhance the $\mathrm{z}$ polarized component of the excitation and detection [10].

Theta microscopy offers another possibility: as the illumination and detection axes are crossed, the optical axis for the detection arm corresponds to a lateral axis of the illumination arm, for which the component is expected to be of much higher intensity. As shown on Figure 1, choosing a x'- or y'-polarized illumination wave corresponds to enhancing detection for the $\mathrm{x}$ - or $\mathrm{z}$ - component, respectively. The equations giving the illumination field, therefore the dipole moment, are readily obtained from Eqs. (1-2) by a $90^{\circ}$ rotation around the $\left(z^{\prime}=-y\right)$-axis, thus permuting the roles of the variable $\mathrm{x}$ and $-\mathrm{z}$. Figure $2(\mathrm{c})$ shows the PSF obtained for z-polarized excitation and x-polarized detection. Note that the maxima of the PSFs are the same in x-polarized or z-polarized excitation. Polarized theta microscopy may therefore constitute an interesting alternative to z-polarized confocal microscopy for large specimen [10].

Figure 3 shows PSFs computed for crossed polars, with y-polarized detection. Not only are the shapes different, but also the relative intensities of the PSFs, the detection for crossed-polarization being typically 400 times weaker. Figure 3(a) shows the computed PSF whith x-polarized excitation and ypolarized detection (in the $\mathrm{z}=0 \mu \mathrm{m}$ and $\mathrm{y}=0 \mu \mathrm{m}$ planes, respectively). It exhibits the characteristic fourleave pattern also observed in confocal polarized microscopy $[6,7,9,11]$, but now in both planes. The pattern is however now asymetric along the z-axis, as can be seen on Figure 3(b), showing (x-z) cuts along the $\mathrm{y}=-0.18 \mu \mathrm{m}$ and $\mathrm{y}=+0.18 \mu \mathrm{m}$, respectively. Figure 3(c) shows the PSF computed for $\mathrm{z}$-polarized excitation and $\mathrm{y}$-polarized detection (in the $\mathrm{z}=0 \mu \mathrm{m}$ and $\mathrm{y}=0 \mu \mathrm{m}$ planes, respectively). The PSF has now a very different shape, as can be seen on Figures 3(d) and 3(c), which show cuts along various y- and zplanes. Note that a central symmetry is conserved.

To favor the y-polarized component, one could either symetryze the detection and illumination arms, or use a second illumination objective, placed perpendicularly to the theta setup (along the x-axis), in a configuration similar to Multiple Objective Microscopy [12] or Multiple Imaging Axis Microscopy [13].

\section{Conclusion}

We have given a rigorous electromagnetic treatment of polarized confocal theta microscopy, using the dipole emission model to describe the fluorescence process. Formulas to compute Point Spread Functions for this type of microscopy are given as well as typical examples of PSF. The dipole emission model predicts a slightly more isotropic resolution than the isotropic emission one. The main advantage is the possibility of using strongly z-polarized excitation. Taking into account polarization properties of the fluorescence emission may permit to obtain additional information about the distribution and orientation of the dipoles in large specimen with a good spatial resolution.

This work was made possible with the financial support of the New Energy and Industrial Technology Development Organization (NEDO) of Japan under an Industrial Technology Research Grant Program. 


\section{References}

1. Richards B. and Wolf E., Proc. R. Soc. London Ser. A 253 (1959) 349

2. Minsky M., Scanning 10 (1988) 128

3. Stelzer E. H. K. and Lindek S., Opt. Commun. 111 (1994) 536

4. Perrin F., Ann. Phys. (Paris) 12 (1929) 169

5. Soleillet P., Ann. Phys. (Paris) 12, (1929) 23

6. Török P., Higdon P.D. and Wilson T., Opt. Comm. 148 (1998) 12

7. Török P., Higdon P.D. and Wilson T., J. Mod. Opt. 45 (1998) 1681

8. Olympus France (private communication)

9. Sheppard C.J.R and Török P, Bioimaging 5 (1997) 205

10. Huse N., Schönle A. and Hell S.W., J. Biomed. Opt. 6 (2001) 480

11. Wilson T., Juskaitis R., and Higdon P., Opt. Comm. 141 (1997) 298

12. Haeberlé O., Xu C., Dieterlen A. and Jacquey S., Compt. Rend. Acad. Sc. IV (Paris) 2 (2001) 1509

13. Stelzer E.H.K., Swoger J. and Huisken J., "Multiple imaging axis microscopie (MIAM)", in Technical Digest of Focus on Microscopy 2002 (Kaohsiung, 2002), p. 58 


\section{Figure Captions}

Figure 1: Sketch of a confocal theta microscope. Objective 1 is used for sample excitation. Objective 2, with optical axis at $90^{\circ}$ with respect to objective 1 , is used for detecting the fluorescence signal.

Figure 1 : Schéma de principe d'un microscope confocal theta. L'objectif 1 sert à l'excitation du specimen. L'objectif 2, dont l'axe optique est à $90^{\circ}$ de l'objectif 1 , sert à la detection du signal de fluorescence

Figure 2: Point Spread Functions for a theta microscope using water immersion objectives with NA=0.8, excitation at $\lambda=400 \mathrm{~nm}$ and detection at $\lambda=450 \mathrm{~nm}$ (Cascade Blue - Molecular Probes). (a) conventional unpolarized isotropic radiation model. (b-c) dipole emission model. (b) x-polarized excitation, x-polarized detection. (c) z-polarized excitation, x-polarized detection.

Figure 2 : Reponse Impulsionelle Optique pour un microscope theta utilisant des objectifs à immersion à eau (N.A. $=0.8$ ). Excitation à $\lambda=400 \mathrm{~nm}$ et détection à $\lambda=450 \mathrm{~nm}$ (Cascade Blue - Molecular Probes). (a) modèle classique d'émission isotrope non polarisée. (b-c) modèle dipolaire. (b) excitation x-polarisée, détection x-polarisée. (c) excitation z-polarisée, détection x-polarisée.

Figure 3: Same as Figure 2, but for y-polarized detection. (a) x-polarized excitation, y-polarized detection. (b) same as (a)-bottom, but in the $y=-0.18 \mu \mathrm{m}$ and $\mathrm{y}=+0.18 \mu \mathrm{m}$ plane respectively. Note the assymetry. (c) z-polarized excitation, y-polarized detection. (d-e) same as (c) but in the $z= \pm 0.15 \mu \mathrm{m}$ and $\mathrm{y}= \pm 0.15 \mu \mathrm{m}$ plane. Note the complex shape of the PSF in that case.

Figure 3 : Idem à Figure 2, mais pour une détection y-polarisée. (a) excitation x-polarisée, détection ypolarisée. (b) identique à (a)-bas dans les plans $\mathrm{y}=-0.18 \mu \mathrm{m}$ et $\mathrm{y}=+0.18 \mu \mathrm{m}$. Notez l'assymétrie. (c) excitation z-polarisée, détection y-polarisée. (d-e) identique à (c) mais dans les plans $\mathrm{z}= \pm 0.15 \mu \mathrm{m}$ et $\mathrm{y}= \pm 0.15 \mu \mathrm{m}$. Notez la forme complexe de la RIO dans ce cas. 


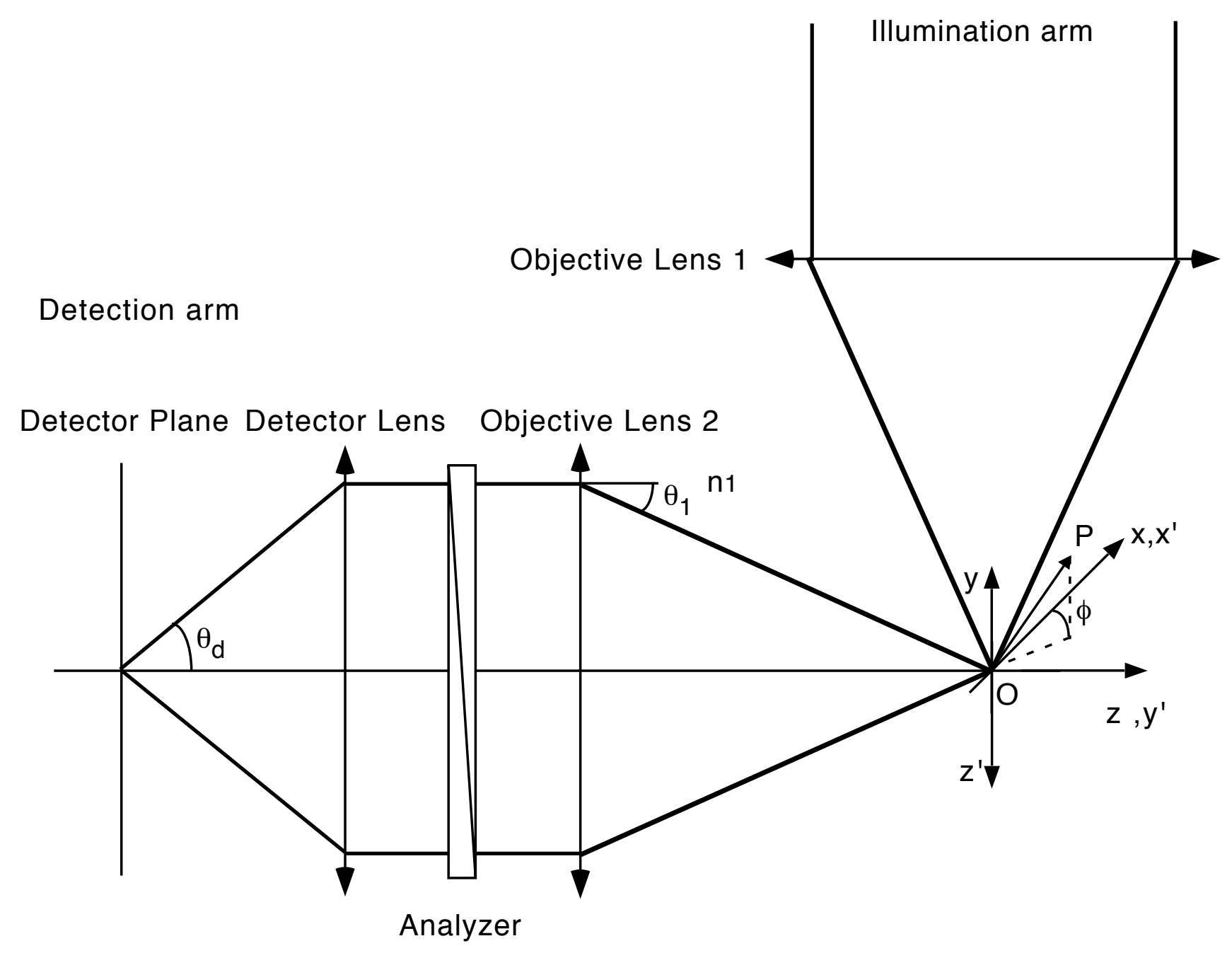

Figure 1 


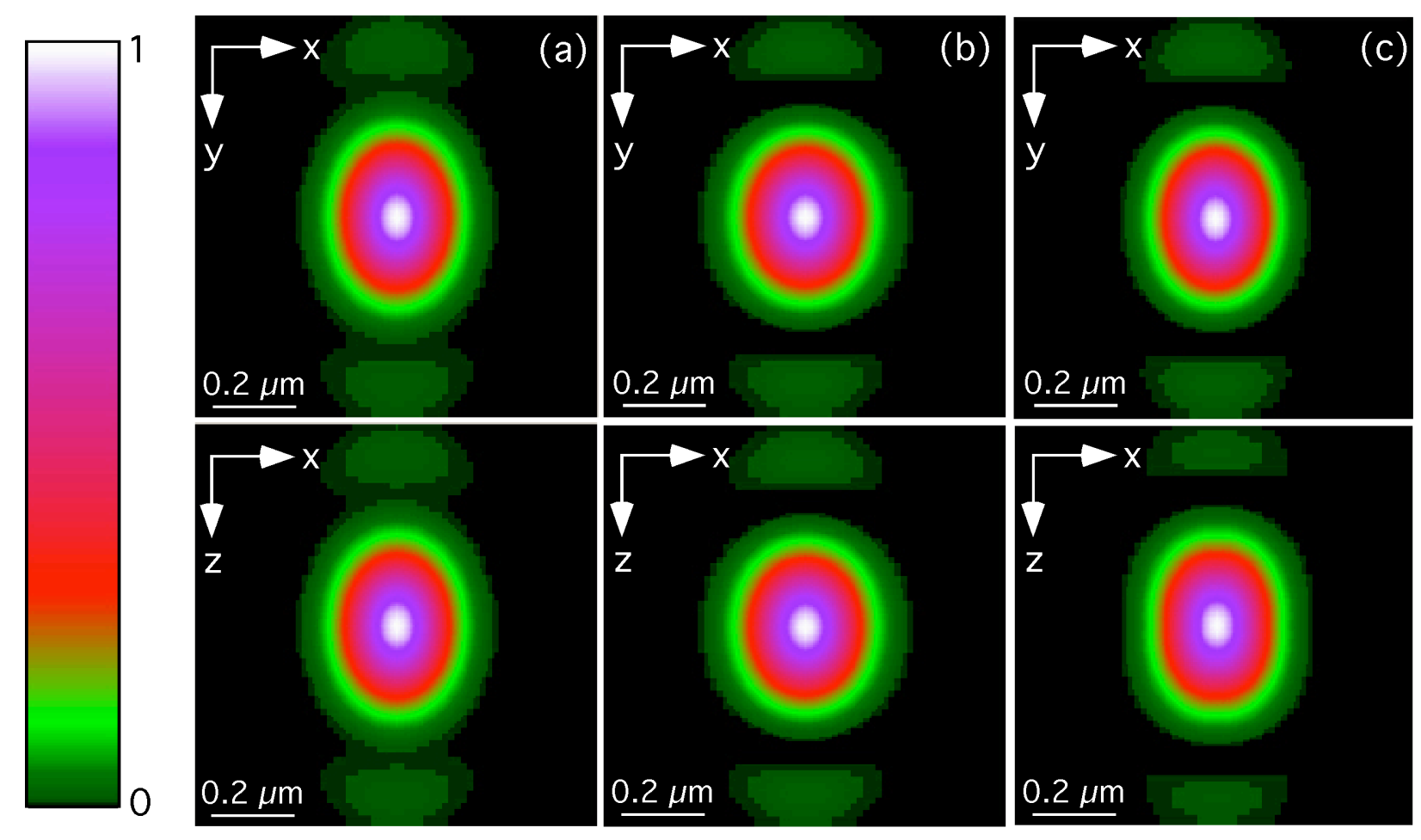

Figure 2 


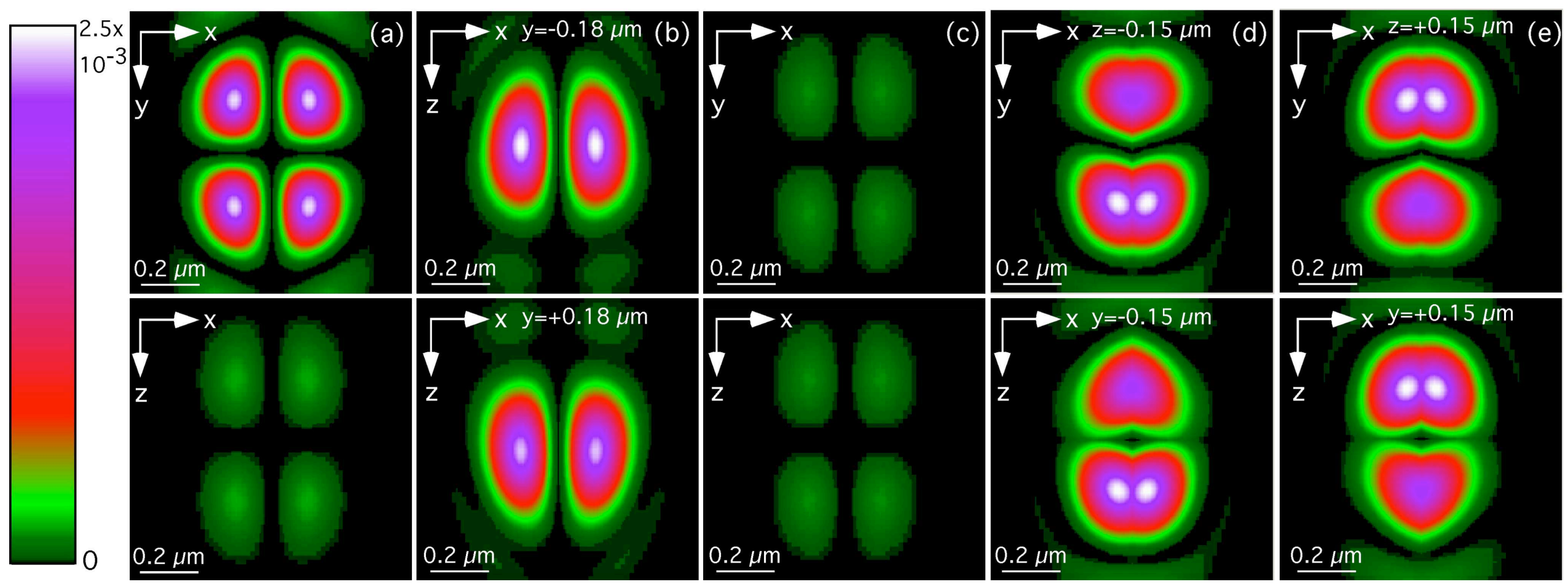

Figure 3 